\title{
Hydrogen sulfide attenuates doxorubicin-induced cardiotoxicity by inhibition of the p38 MAPK pathway in H9c2 cells
}

\author{
RUNMIN GUO ${ }^{1}$, JIANCONG LIN ${ }^{2}$, WENMING XU $^{2}$, NING SHEN $^{1}$, \\ LIQIU MO ${ }^{3}$, CHANGRAN ZHANG ${ }^{2}$ and JIANQIANG FENG ${ }^{1}$
}

${ }^{1}$ Department of Physiology, Zhongshan School of Medicine, Sun Yat-sen University; Departments of ${ }^{2}$ Internal Medicine and
${ }^{3}$ Anesthesiology, Huangpu Division of The First Affiliated Hospital, Sun Yat-sen University, Guangzhou 510080, P.R. China

Received October 21, 2012; Accepted December 4, 2012

DOI: $10.3892 /$ ijmm.2013.1246

\begin{abstract}
We previously demonstrated the protective effect of hydrogen sulfide $\left(\mathrm{H}_{2} \mathrm{~S}\right)$ against doxorubicin (DOX)-induced cardiotoxicity through inhibition of endoplasmic reticulum stress. The aim of the present study was to explore the role of p38 mitogen-activated protein kinase (MAPK) in DOX-induced cardiotoxicity and ascertain whether exogenous $\mathrm{H}_{2} \mathrm{~S}$ protects DOX-induced injury by inhibiting p38 MAPK in cardiomyoblasts (H9c2). We observed that exposure of H9c2 cells to $5 \mu \mathrm{M}$ DOX not only markedly induced injuries, including cytotoxicity, apoptosis, overproduction of reactive oxygen species (ROS) and dissipation of mitochondrial membrane potential (MMP), but also enhanced the expression level of phosphorylated (p)-p38 MAPK. The DOX-induced increase in expression of p-p38 MAPK was significantly attenuated by pretreatment of $\mathrm{H} 9 \mathrm{c} 2$ cells with either $400 \mu \mathrm{M}$ sodium hydrogen sulfide (NaHS) (a donor of $\mathrm{H}_{2} \mathrm{~S}$ ) or $1,000 \mu \mathrm{M} \mathrm{N}$-acetyl-L-cysteine (NAC, an ROS scavenger) prior to exposure to DOX. Pretreatment with either $400 \mu \mathrm{M}$ NaHS or $3 \mu \mathrm{M} \mathrm{SB} 203580$, a selective inhibitor of p38 MAPK, ameliorated DOX-induced cardiomyocyte injuries, as evidenced by an increase in cell viability, and decreases in the number of apoptotic cells, ROS generation as well as dissipation of MMP. In conclusion, the findings of the present study demonstrated that the activation of p38 MAPK contributes to DOX-induced injuries, including cytotoxicity, apoptosis, mitochondrial damage and oxidative stress in $\mathrm{H} 9 \mathrm{c} 2$ cells. We also provide novel evidence that exogenous $\mathrm{H}_{2} \mathrm{~S}$ protects $\mathrm{H} 9 \mathrm{c} 2$ cells against DOX-induced cardiotoxicity by inhibition of the p38 MAPK pathway.
\end{abstract}

Correspondence to: Professor Changran Zhang, Department of Internal Medicine, Huangpu Division of the First Affiliated Hospital, Sun Yat-sen University, Guangzhou 510080, P.R. China E-mail: zhangchangran@yeah.net

Professor Jianqiang Feng, Department of Physiology, Zhongshan School of Medicine, Sun Yat-sen University, Guangzhou 510080, P.R. China

E-mail: fengjq-sums@163.com

Key words: hydrogen sulfide, doxorubicin, cardiotoxicity, p38 mitogen-activated protein kinase, $\mathrm{H} 9 \mathrm{c} 2$ cells

\section{Introduction}

To date, doxorubicin (DOX) remains one of the most widely used antitumor agents (1). It is a valuable component of various chemotherapeutic regimens used to treat breast and small-cell lung carcinoma as well as leukemia. However, its clinical use is limited by severe, dose-dependent acute and chronic cardiotoxicity $(2,3)$, which may ultimately lead to severe and irreversible cardiomyopathy (4). The cause of DOX-induced cardiotoxicity is multifactorial, even though most of DOX-elicited cardiac effects are contributed to reactive oxygen species (ROS) generation, which ultimately leads to cardiomyocyte apoptosis $(5,6)$. The signal transduction pathway that links DOX-induced oxidative stress and cardiac injury is a topic of strong current interest. Increasing evidence reveals that p38 mitogen-activated protein kinase (MAPK), one of the members of the MAPK family, may play an important role in DOX-induced cardiotoxicity $(7,8)$.

p38 MAPK is activated by cellular stress and is thought to participate in cardiomyocyte apoptosis and cardiac pathologies $(9,10)$. In isolated cardiomyocytes, DOX induces activation of $\mathrm{p} 38 \mathrm{MAPK}$, followed by activation of pro-apoptotic protein Bax (11). In a previous in vivo study, multiple treatments of DOX to rats resulted in a persistent increase in the phosphorylation of p38 MAPK (12). The results of these studies $(11,12)$ suggested that the p38 MAPK pathway may be involved in DOX-induced cardiotoxicity. However, the role of the p38 MAPK pathway in DOX-induced cardiotoxicity is not completely understood.

More recently, we demonstrated that hydrogen sulfide $\left(\mathrm{H}_{2} \mathrm{~S}\right)$ protects against DOX-induced cardiotoxicity through inhibition of endoplasmic reticulum (ER) stress and oxidative stress (13). $\mathrm{H}_{2} \mathrm{~S}$, a well-known toxic gas with the characteristic smell of rotten eggs, has been qualified as the third gasotransmitter along with nitric oxide (NO) and carbon monoxide (CO) $(14,15)$. Accumulating evidence has shown that $\mathrm{H}_{2} \mathrm{~S}$ plays an important physiologic and pathophysiological role in regulating cardiovascular function. Exogenous $\mathrm{H}_{2} \mathrm{~S}$ can attenuate myocardial necrosis and rescue contractile activity in isoproterenol-stimulated rat hearts (16). $\mathrm{H}_{2} \mathrm{~S}$ also ameliorates hyperhomocysteinemia-induced myocardial tissue damage by inhibiting ER stress-associated apoptosis in rats (17). Recently, we demonstrated that exogenous $\mathrm{H}_{2} \mathrm{~S}$ not 
only protects H9c2 cells from hypoxia-induced injury (18), but also inhibits hypoxia-induced activation of p38 MAPK (19). Kang et al (7) also reported that metallothionein (MT) which functions as an antioxidant can suppress DOX-induced apoptosis by inhibition of p38 MAPK. Based on our recent studies $(18,19)$ and a previous one $(20)$, we speculate that inhibition of the p38 MAPK pathway may contribute to the protective effect of exogenous $\mathrm{H}_{2} \mathrm{~S}$ against DOX-induced cardiotoxicity.

To test this hypothesis, in the present study, H9c2 cells were treated with $5 \mu \mathrm{M}$ DOX to establish a chemotherapyinduced cardiotoxicity model (13). We then explored i) the effect of DOX on the phosphorylation of p38 MAPK; ii) the effect of exogenous $\mathrm{H}_{2} \mathrm{~S}$ on DOX-induced increase in the activation of p38 MAPK; iii) the role of the activation of p38 MAPK in DOX-induced cardiomyocyte insults; and iv) whether exogenous $\mathrm{H}_{2} \mathrm{~S}$ protects $\mathrm{H} 9 \mathrm{c} 2$ cells against DOX-induced cardiotoxicity by inhibiting the p38 MAPK pathway.

\section{Materials and methods}

Materials. Sodium hydrogen sulfide (NaHS), SB203580, DOX, dichlorofluorescein diacetate (DCFH-DA) and Hoechst 33258 were purchased from Sigma-Aldrich (St. Louis, MO, USA). The Cell Counting Kit-8 (CCK-8) was purchased from Dojindo Lab (Japan). JC-1 was purchased from Molecular Probes. DMEM-F12 medium and fetal bovine serum (FBS) were purchased from Gibco-BRL. H9c2 cells were obtained from the Sun Yat-sen University Experimental Animal Center.

Cell culture and treatments. $\mathrm{H} 9 \mathrm{c} 2$ cells were cultured in DMEM-F12 medium supplemented with $10 \% \mathrm{FBS}$ at $37^{\circ} \mathrm{C}$ under an atmosphere of $5 \% \mathrm{CO}_{2}$. To explore the protective effects of $\mathrm{H}_{2} \mathrm{~S}$ on DOX-induced injury, $\mathrm{H} 9 \mathrm{c} 2$ cells were pretreated with NaHS (a well-known $\mathrm{H}_{2} \mathrm{~S}$ donor) for $30 \mathrm{~min}$ prior to DOX treatment. To further determine whether the protective effects of $\mathrm{H}_{2} \mathrm{~S}$ were associated with the inhibition of p38 MAPK activity, H9c2 cells were pretreated with SB203580, a selective inhibitor of p38 MAPK, for $60 \mathrm{~min}$ prior to DOX treatment.

Cell viability assay. After H9c2 cells cultured in 96-well plates received different treatments, $10 \mu \mathrm{l} \mathrm{CCK-8} \mathrm{solution} \mathrm{was}$ added to each well at a $1 / 10$ dilution, followed by a further 2-h incubation in the incubator. Absorbance was measured at $450 \mathrm{~nm}$ with a microplate reader (Multiskan MK3 Microplate Reader, Thermo Fisher Scientific, Inc., USA). The mean optical density (OD) of 5 wells in the indicated groups was used to calculate the percentage of cell viability according to the formula:

Percentage of cell viability $=$ OD treatment group/OD control group $\mathrm{x} 100 \%$. Experiments were performed in triplicate.

Hoechst 33258 nuclear staining to assess apoptosis. Apoptosis was analyzed by fluorescence microscopy with the chromatin dye Hoechst 33258. H9c2 cells were analyzed $24 \mathrm{~h}$ following the indicated treatments. Cells were fixed with $4 \%$ paraformaldehyde in phosphate-buffered saline (PBS) for $10 \mathrm{~min}$. Following 3 washes with PBS, cells were stained with $5 \mathrm{mg} / \mathrm{l}$ Hoechst 33258 for $5 \mathrm{~min}$, washed briefly with PBS and air-dried again. The cells were visualized under a fluorescence microscope (BX50-FLA; Olympus, Tokyo, Japan). Apoptotic cells showed condensed, fractured or distorted nuclei, viable cells displayed normal nuclear size and uniform fluorescence.

Measurement of intracellular ROS generation. Intracellular ROS generation was determined by the oxidative conversion of cell-permeable DCFH-DA to fluorescent DCF. H9c2 cells were cultured on a slide in DMEM-F12 medium. After the indicated treatments, slides were washed twice with PBS. DCFH-DA solution in serum-free medium was added at a concentration of $10 \mu \mathrm{M}$ and co-incubated with H9c2 cells at $37^{\circ} \mathrm{C}$ for $60 \mathrm{~min}$. The slides were washed 3 times, and DCF fluorescence was measured over the entire field of vision using a fluorescence microscope connected to an imaging system (BX50-FLA; Olympus). The mean fluorescence intensity (MFI) from 4 random fields was analyzed using ImageJ 1.410 software (National Institutes of Health, USA) and the MFI of DCF was used to indicate the amount of ROS.

Assessment of MMP. Mitochondrial membrane potential (MMP) was assessed using the fluorescent indicator 5,5',6,6'-tetrachloro-1,1'3,3'-tetraethylbenzimidazolocarbocyanine iodide (JC-1; Molecular Probes). The DOX-treated cells were incubated with $1 \mathrm{mg} / \mathrm{ml} \mathrm{JC}-1$ for $30 \mathrm{~min}$ at $37^{\circ} \mathrm{C}$ and visualized using a fluorescence microscope. A shift from red to green fluorescence indicates a loss of MMP, which was assessed by obtaining multiple merged images and using MetaMorph software to count cells that fluoresced red or green. Data are expressed as the percentage of cells that had undergone mitochondrial membrane transition (i.e., gained green fluorescence).

Western blot assay. After different treatments, H9c2 cells were harvested and lysed, and the homogenate was centrifuged at $12,000 \mathrm{rpm}$ for $10 \mathrm{~min}$ at $4^{\circ} \mathrm{C}$. The total protein in the supernatant was quantitated with a BCA protein assay kit. Total protein (30 $\mu \mathrm{g}$ from each sample) was separated by $12 \%$ SDS-PAGE. The protein in the gel was transferred to a polyvinylidene difluoride (PVDF) membrane. The membrane was blocked with 5\% free-fat milk in TBS-T for $1 \mathrm{~h}$ at room temperature, and then incubated with primary antibodies specific to p38 MAPK and phosphorylated (p)-p38 MAPK $(1: 4,000)$, caspase-3 $(1: 2,000)$ (Cell Signaling Technology, Inc., Beverly, MA, USA), or GAPDH with gentle agitation at $4^{\circ} \mathrm{C}$ overnight and subsequently incubated with the secondary antibodies for $1.5 \mathrm{~h}$ at room temperature. Following 3 washes with TBS-T, membranes were developed using enhanced chemiluminescence and exposed to X-ray films. To quantify protein expression, the X-ray films were scanned and analyzed with ImageJ 1.41 o software.

Statistical analysis. All data are presented as means \pm standard error (SE). Differences between groups were analyzed by one-way analysis of variance (ANOVA) with SPSS 13.0 (SPSS Inc., Chicago, IL, USA). $\mathrm{P}<0.05$ was considered to indicate a statistical significant difference. 
A

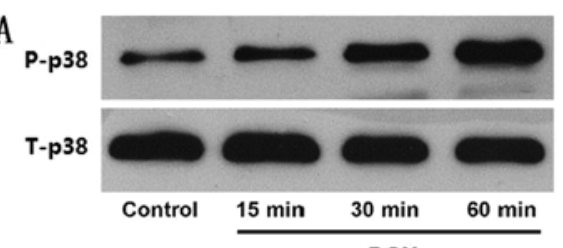

B

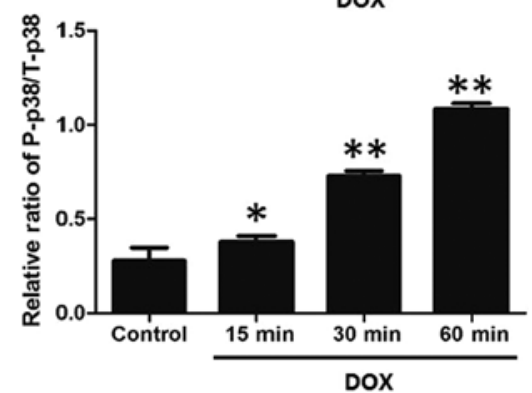

Figure 1. DOX time-dependently increases the expression of phosphorylated (P)-p38 MAPK in H9c2 cells. H9c2 cells were treated with $5 \mu \mathrm{M}$ for the indicated times. (A) Expression of p38 MAPK was assessed by western blot analysis and (B) quantified by densitometric analysis with ImageJ 1.410 software. Data are shown as the means $\pm \mathrm{SE}(\mathrm{n}=3) .{ }^{*} \mathrm{P}<0.05,{ }^{* * *} \mathrm{P}<0.01$ vs. the control group. T, total. DOX, doxorubicin.
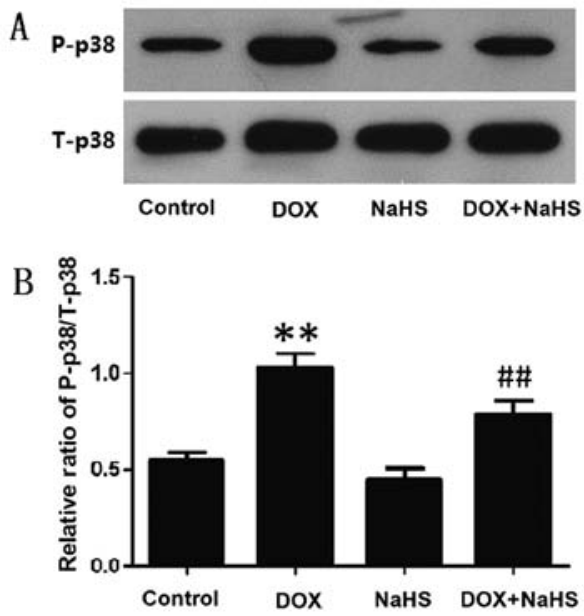

Figure 2. Exogenous $\mathrm{H}_{2} \mathrm{~S}$ attenuates DOX-induced expression of phosphorylated (P)-p38 MAPK in $\mathrm{H} 9 \mathrm{c} 2$ cells. $\mathrm{H} 9 \mathrm{c} 2$ cells were treated with $5 \mu \mathrm{mol} / 1$ DOX for $60 \mathrm{~min}$ and either untreated or pretreated with $400 \mu \mathrm{mol} / 1 \mathrm{NaHS}$ for 30 min prior to DOX exposure. (A) Expression of p38 MAPK was examined by western blot analysis and (B) quantified by densitometric analysis with ImageJ 1.41 o software. Data are shown as the means $\pm \mathrm{SE}$ $(\mathrm{n}=3) .{ }^{* *} \mathrm{P}<0.01$ vs. the control group; ${ }^{\# \#} \mathrm{P}<0.01$ vs. the DOX-treated group. $\mathrm{T}$, total; DOX, doxorubicin.

\section{Results}

DOX increases the expression of phosphorylated p38 MAPK in $\mathrm{H} 9 \mathrm{c} 2$ cells. To test the effect of DOX on activation of p38 MAPK, H9c2 cells were treated with $5 \mu \mathrm{M}$ DOX for the indicated times (15, 30 and $60 \mathrm{~min})$. As shown in Fig. 1, DOX time-dependently enhanced the expression level of p-p38 MAPK. However, DOX at $5 \mu \mathrm{M}$ alone did not induce significant changes in expression of total (t) p38 MAPK.

Exogenous $\mathrm{H}_{2} \mathrm{~S}$ inhibits DOX-induced increase in expression of phosphorylated p38 MAPK in H9c2 cells. Western

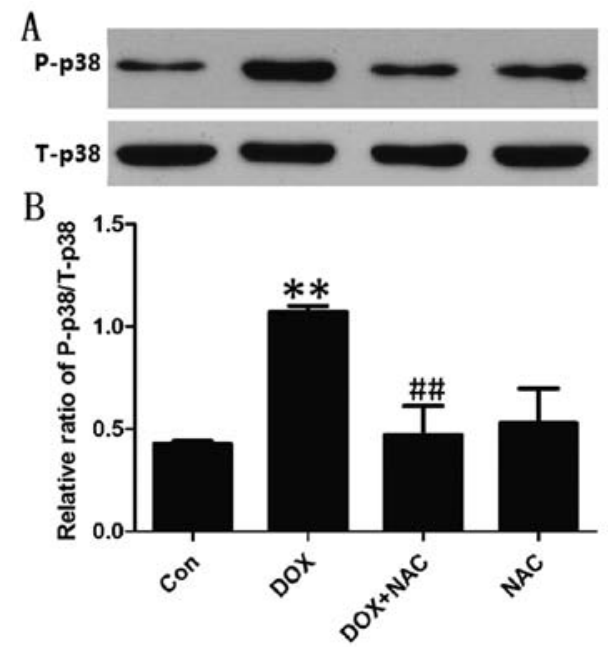

Figure 3. NAC suppresses DOX-induced activation of p38 MAPK in H9c2 cells. H9c2 cells were treated with $5 \mu \mathrm{mol} / 1 \mathrm{DOX}$ for $60 \mathrm{~min}$ and either treated or pretreated with $1,000 \mu \mathrm{mol} / 1 \mathrm{NAC}$ for $60 \mathrm{~min}$. (A) Western blot analysis was applied to detect change in expression of total (T)-p38 MAPK and phosphorylated (P)-p38 MAPK. (B) Densitometric analysis of the results in $\mathrm{A}$. The data are presented as the means $\pm \mathrm{SE}(\mathrm{n}=3) .{ }^{* *} \mathrm{P}<0.01$ vs. the control group; ${ }^{\# \#} \mathrm{P}<0.01$ vs. the DOX treatment group. DOX, doxorubicin.

blot analysis showed that exposure of $\mathrm{H} 9 \mathrm{c} 2$ cells to $5 \mu \mathrm{M}$ DOX for 60 min markedly enhanced the expression level of p-p38 MAPK (Fig. 2A and B). This increased expression of p-p38 MAPK was attenuated by pretreatment of cells with $400 \mu \mathrm{M}$ NaHS (a donor of $\mathrm{H}_{2} \mathrm{~S}$ ) for 30 min prior to exposure to $5 \mu \mathrm{M}$ DOX. NaHS at $400 \mu \mathrm{M}$ alone did not alter the basal expression level of p-p38 MAPK in H9c2 cells (Fig. 2A and B).

NAC suppresses the DOX-induced activation of p38 MAPK in $H 9 c 2$ cells. To confirm whether the inhibitory effect of NaHS on the DOX-induced increase in expression of p-p38 MAPK is associated with its antioxidation, H9c2 cells were pretreated with $1,000 \mu \mathrm{M}$ NAC (ROS scavenger) for $60 \mathrm{~min}$ before exposure to $5 \mu \mathrm{M}$ DOX. As shown in Fig. 3, similar to the inhibitory effect of NaHS pretreatment, the pretreatment of cells with NAC for 60 min markedly depressed the increased expression of p-p38 MAPK induced by $5 \mu \mathrm{M}$ DOX for $60 \mathrm{~min}$. NAC at $1,000 \mu \mathrm{M}$ did not significantly change the basal level of p-p38 MAPK expression. The results revealed that an antioxidant effect contributed to the inhibitory effect of $\mathrm{H}_{2} \mathrm{~S}$ on the DOX-induced increased expression of p-p38 MAPK.

Exogenous $\mathrm{H}_{2} \mathrm{~S}$ and p38 MAPK inhibitor attenuate DOX-induced cytotoxicity in $\mathrm{H} 9 \mathrm{c} 2$ cells. As presented in Fig. 4 , exposure of $\mathrm{H} 9 \mathrm{c} 2$ cells to DOX at $5 \mu \mathrm{M}$ for $24 \mathrm{~h}$ induced marked cytotoxicity, leading to a decrease in cell viability. However, pretreatment of cells with $400 \mu \mathrm{M}$ NaHS for 30 min prior to exposure to DOX significantly ameliorated the DOX-induced cytotoxicity, as evidenced by an increase in cell viability. In order to examine whether the activation of p38 MAPK is involved in DOX-induced cytotoxicity, H9c2 cells were pretreated with $3 \mu \mathrm{M} \mathrm{SB} 203580$, a selective inhibitor of p38 MAPK, for $60 \mathrm{~min}$ before exposure to $5 \mu \mathrm{M}$ 


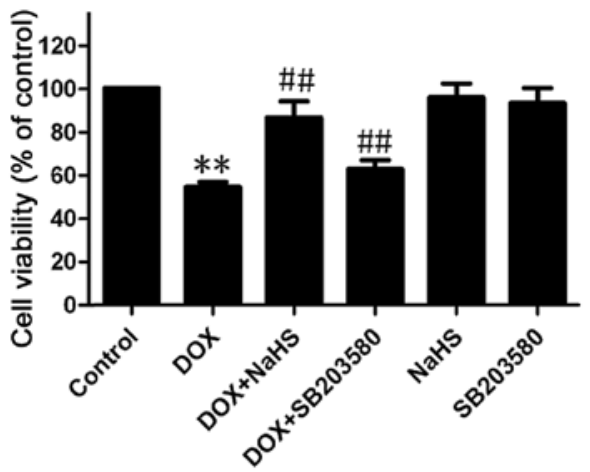

Figure 4. Exogenous $\mathrm{H}_{2} \mathrm{~S}$ and p38 MAPK inhibitor protect $\mathrm{H} 9 \mathrm{c} 2$ cells against DOX-induced cytotoxicity. H9c2 cells were treated with $5 \mu \mathrm{mol} / 1$ DOX for $24 \mathrm{~h}$ in the absence of or pretreated with $400 \mu \mathrm{M} \mathrm{NaHS}$ for $30 \mathrm{~min}$ or $3 \mu \mathrm{M} \mathrm{SB} 203580$ for $60 \mathrm{~min}$ before DOX treatment. Cell viability was measured using the CCK- 8 assay. Data are shown as the means $\pm \operatorname{SE}(n=3)$. ${ }^{* *} \mathrm{P}<0.01$ vs. the control group; ${ }^{\# \#} \mathrm{P}<0.01$ vs. the DOX treatment group. DOX, doxorubicin.
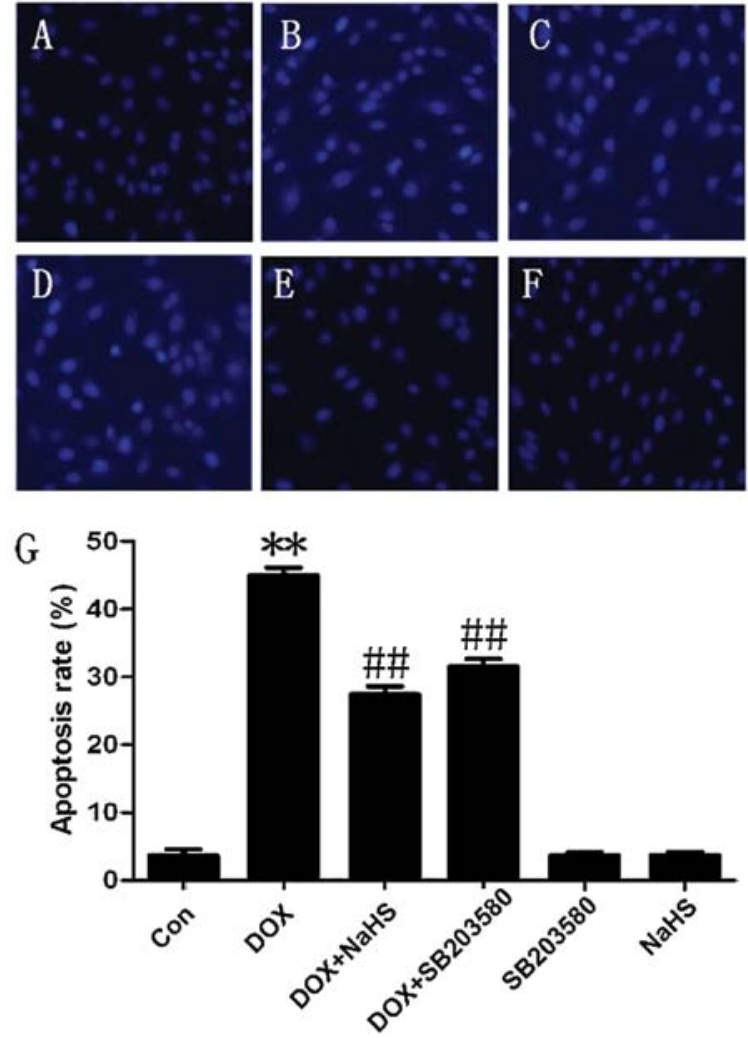

Figure 5. Exogenous $\mathrm{H}_{2} \mathrm{~S}$ and p38 MAPK inhibitor reduce DOX-induced apoptosis in H9c2 cells. (A-F) Hoechst 33258 nuclear staining followed by fluorescence imaging to observe cell apoptosis. (A) Control group; H9c2 cells were (B) exposed to $5 \mu \mathrm{M}$ DOX for $24 \mathrm{~h}$; (C) pretreated with $400 \mu \mathrm{M}$ NaHS for $30 \mathrm{~min}$ prior to exposure to $5 \mu \mathrm{M}$ DOX for $24 \mathrm{~h}$; (D) treated with $3 \mu \mathrm{M} \mathrm{SB} 203580$ for $60 \mathrm{~min}$ followed by exposure to $5 \mu \mathrm{M}$ DOX for $24 \mathrm{~h}$; (E) treated with $400 \mu \mathrm{M}$ NaHS for 30 min followed by $24 \mathrm{~h}$ culture; (F) treated with $3 \mu \mathrm{M}$ SB203580 for 60 min followed by 24 -h culture. (G) The apoptotic rate was analyzed with a cell counter and ImageJ 1.41 software. ${ }^{* *} \mathrm{P}<0.01$ vs. the control group; ${ }^{\# \#} \mathrm{P}<0.01$ vs. the DOX treatment group. DOX, doxorubicin.

DOX for $24 \mathrm{~h}$. Pretreatment with SB203580 had a similar cytoprotective effect as $\mathrm{H}_{2} \mathrm{~S}$ against DOX-induced cytotoxicity, suggesting involvement of the activation of p38 MAPK

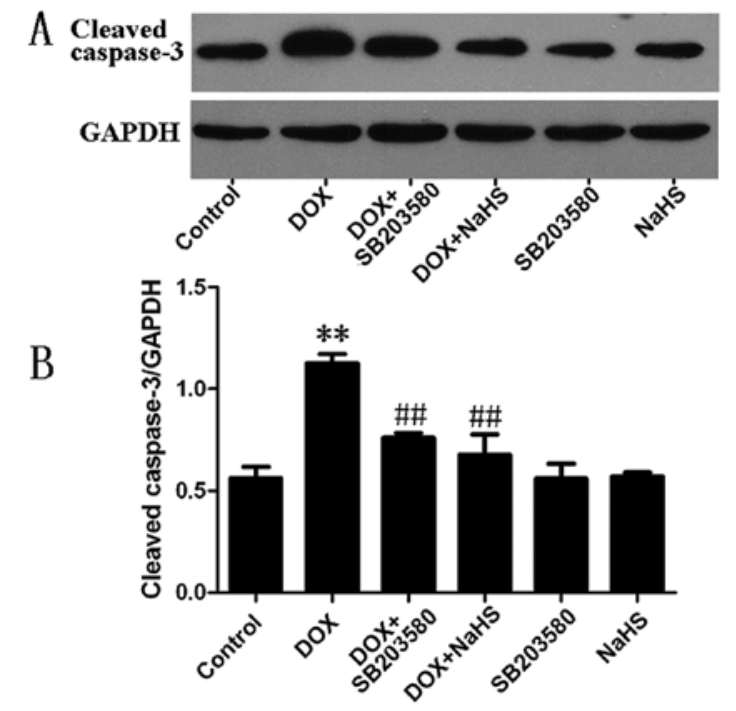

Figure 6. Exogenous $\mathrm{H}_{2} \mathrm{~S}$ and p38 MAPK inhibitor suppress the DOXinduced increase in the expression of cleaved caspase- 3 in $\mathrm{H} 9 \mathrm{c} 2$ cells. H9c2 cells were treated with $5 \mu \mathrm{mol} / 1 \mathrm{DOX}$ for $12 \mathrm{~h}$ in the absence of pretreated with $400 \mu \mathrm{M}$ NaHS for $30 \mathrm{~min}$ or $3 \mu \mathrm{M} \mathrm{SB} 203580$ for $60 \mathrm{~min}$ prior to DOX exposure. (A) Expression of cleaved caspase-3 was assessed by western blot assay. (B) The data in (A) were quantified by densitometric analysis with ImageJ 1.41 software. Data are shown as the means $\pm \mathrm{SE}(\mathrm{n}=3){ }^{*}{ }^{* *} \mathrm{P}<0.01$ vs. the control group; ${ }^{\# \#} \mathrm{P}<0.01$ vs. the DOX-treated group. DOX, doxorubicin.

in DOX-induced cytotoxicity in H9c2 cells. Alone NaHS or SB203580 did not alter cell viability in the H9c2 cells (Fig. 4).

Exogenous $\mathrm{H}_{2} \mathrm{~S}$ and p38 MAPK inhibitor reduce DOX-induced apoptosis in $H 9 c 2$ cells. We further observed the effects of both NaHS and p38 MAPK inhibitor on DOX-induced apoptosis. As shown in Fig. 5B, H9c2 cells treated with $5 \mu \mathrm{M}$ for $24 \mathrm{~h}$ exhibited typical characteristics of apoptosis, including condensation of chromatin, shrinkage of nuclei and apoptotic bodies. However, pretreatment of cells with $400 \mu \mathrm{M}$ NaHS for 30 min before DOX exposure obviously decreased the DOX-induced increased number of cells with nuclear condensation and fragmentation (Fig. 5C). NaHS alone did not markedly alter cell morphology or the percentage of apoptotic H9c2 cells (Fig. 5F and G). In addition, the western blot analysis (Fig. 6A and B) revealed that exposure of cells to $5 \mu \mathrm{M}$ DOX for $12 \mathrm{~h}$ considerably upregulated the expression level of cleaved caspase-3 which is thought to be one of the main effectors of apoptosis (21); this effect was dramatically suppressed by pretreatment of cells with $400 \mu \mathrm{M}$ NaHS for 30 min (Fig. 6A and B). NaHS alone did not affect the basal expression of cleaved caspase- 3 in $\mathrm{H} 9 \mathrm{c} 2$ cells. The above findings indicate that exogenous $\mathrm{H}_{2} \mathrm{~S}$ protects $\mathrm{H} 9 \mathrm{c} 2$ cells against DOX-induced apoptosis.

To ascertain whether the activation of p38 MAPK is implicated in DOX-induced apoptosis, H9c2 cells were pretreated with $3 \mu \mathrm{M}$ SB203580 for $60 \mathrm{~min}$ followed by exposure to $5 \mu \mathrm{M}$ DOX for 24 or $12 \mathrm{~h}$ (for examining cleaved caspase-3 expression). The results showed that pretreatment with SB203580 attenuated not only the DOX-induced increased number of apoptotic H9c2 cells (Fig. 5D and G), but also the expression level of cleaved caspase- 3 induced by DOX (Fig. 6). Alone, SB203580 did not induce H9c2 cell apoptosis 

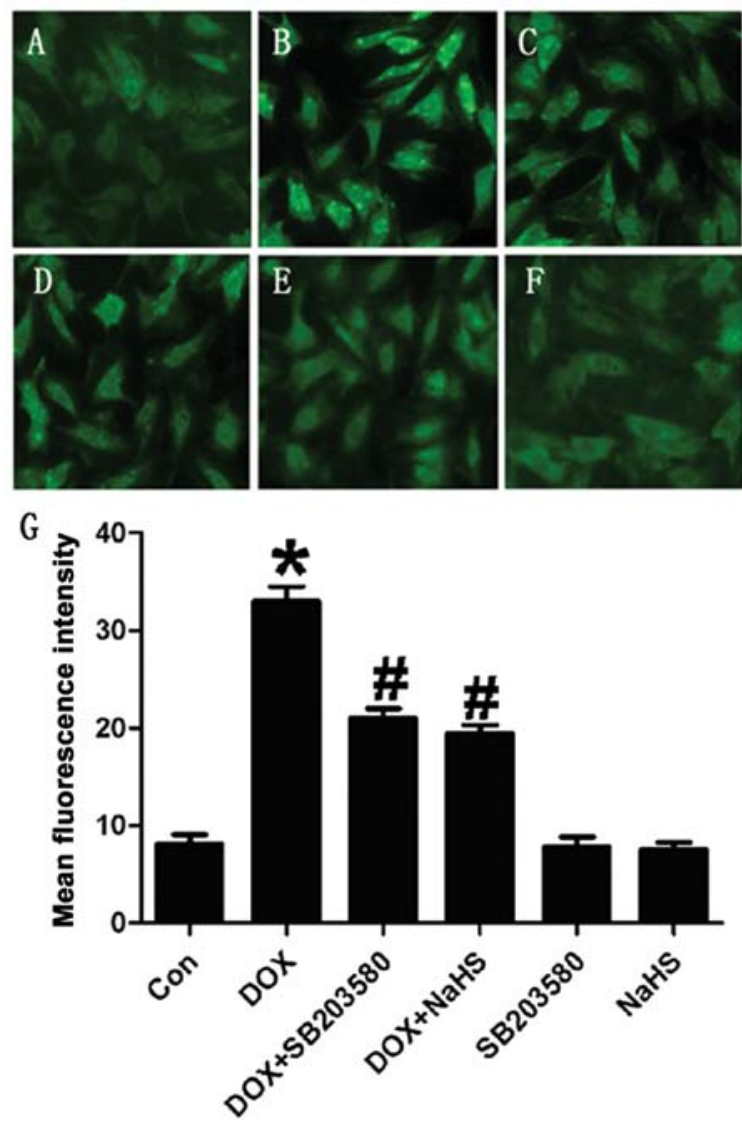

Figure 7. Exogenous $\mathrm{H}_{2} \mathrm{~S}$ and p38 MAPK inhibitor decrease DOX-induced ROS accumulation in $\mathrm{H} 9 \mathrm{c} 2$ cells. (A-F) After the indicated treatments, intracellular ROS generation was measured by DCFH-DA staining followed by photofluorography. (A) Control group; H9c2 cells were (B) exposed to $5 \mu \mathrm{M}$ DOX for $24 \mathrm{~h}$; (C) pretreated with $400 \mu \mathrm{M}$ NaHS for 30 min before exposure to $5 \mu \mathrm{M}$ DOX for $24 \mathrm{~h}$; (D) pretreated with $3 \mu \mathrm{M} \mathrm{SB} 203580$ for $60 \mathrm{~min}$ before exposure to $5 \mu \mathrm{M}$ DOX for $24 \mathrm{~h}$; (E) treated with $400 \mu \mathrm{M}$ NaHS for 30 min followed by $24-\mathrm{h}$ culture; (F) treated with $3 \mu \mathrm{M}$ SB203580 for $60 \mathrm{~min}$ followed by a 24-h culture. (G) Quantitative analysis of the MFI of DCF in A-F with Image $\mathrm{J} 1.410$ software. Data are shown as means \pm SE $(\mathrm{n}=5)$. ${ }^{*} \mathrm{P}<0.05$ vs. control group; ${ }^{\#} \mathrm{P}<0.05$ vs. the DOX-treated group. DOX, doxorubicin; MFI, mean fluorescence intensity.

or a change in the basal expression of cleaved caspase-3. Our findings suggest that the p38 MAPK pathway participates in the DOX-induced apoptosis of $\mathrm{H} 9 \mathrm{c} 2$ cells.

Exogenous $\mathrm{H}_{2} \mathrm{~S}$ and p38 MAPK inhibitor induce DOX-induced oxidative stress in $\mathrm{H} 9 \mathrm{c} 2$ cells. Previous studies have shown that oxidative stress plays a pivotal role in DOX-induced cardiotoxicity $(5,6,13)$. Thus, we investigated the effects of $\mathrm{H}_{2} \mathrm{~S}$ and SB203580 on DOX-induced ROS generation in H9c2 cells. As shown in Fig. 7, exposure of cells to $5 \mu \mathrm{M}$ DOX for $24 \mathrm{~h}$ markedly enhanced ROS generation. The increased ROS generation was attenuated by pretreatment of cells with $400 \mu \mathrm{M}$ NaHS for $30 \mathrm{~min}$ before DOX exposure, suggesting that exogenous $\mathrm{H}_{2} \mathrm{~S}$ protects $\mathrm{H} 9 \mathrm{c} 2$ cells against DOX-induced oxidative stress. To explore whether the activation of $\mathrm{p} 38$ MAPK contributes to the DOX-induced overproduction of ROS, H9c2 cells were preconditioned with $3 \mu \mathrm{M}$ SB203580 for $60 \mathrm{~min}$ prior to exposure to DOX. The preconditioning with SB203580 significantly decreased the DOX-induced increase in ROS generation, indicating the
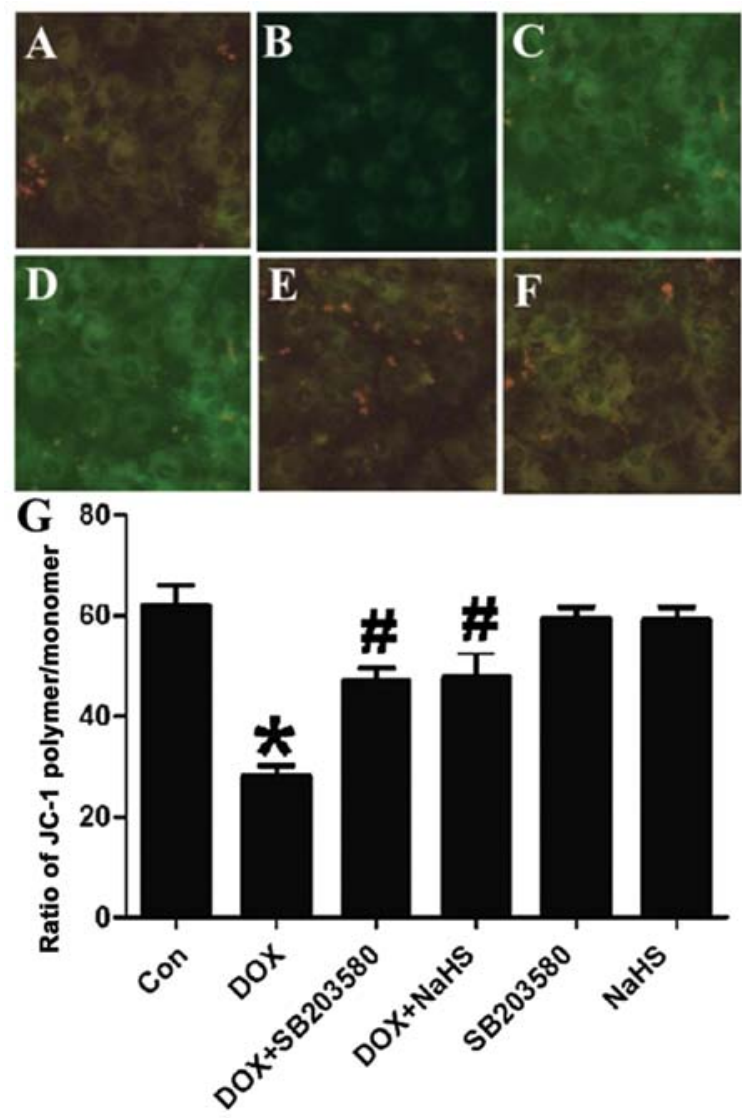

Figure 8. Exogenous $\mathrm{H}_{2} \mathrm{~S}$ and p38 MAPK inhibitor ameliorate DOX-induced MMP loss in H9c2 cells. (A-F) After the indicated treatments, MMP was measured by JC-1 staining followed by photofluorography. (A) Control group; H9c2 cells were (B) treated with $5 \mu \mathrm{M}$ DOX for $24 \mathrm{~h}$; (C) pretreated with $400 \mu \mathrm{M}$ NaHS for $30 \mathrm{~min}$ prior to exposure to $5 \mu \mathrm{M}$ DOX for $24 \mathrm{~h}$; (D) pretreated with $3 \mu \mathrm{M}$ SB203580 for 60 min prior to exposure to $5 \mu \mathrm{M}$ DOX for $24 \mathrm{~h}$; (E) treated with $400 \mu \mathrm{M}$ NaHS for $30 \mathrm{~min}$ followed by a 24-h culture; (F) treated with $3 \mu \mathrm{M}$ SB203580 for 60 min followed by a $24-\mathrm{h}$ culture. (G) Quantitative analysis of the MFI of JC-1 in A-F with Image J 1.41o software. Data are shown as means $\pm \mathrm{SE}, \mathrm{n}=5$. ${ }^{*} \mathrm{P}<0.05$ vs. the control group, ${ }^{\text {}} \mathrm{P}<0.05$ vs. the DOX-treated group. DOX, doxorubicin; MFI, mean fluorescence intensity.

involvement of the p38 MAPK pathway in DOX-induced oxidative stress. NaHS at $400 \mu \mathrm{M}$ or SB203580 at $3 \mu \mathrm{M}$ alone did not alter basal ROS generation (Fig. 7).

Exogenous $\mathrm{H}_{2} \mathrm{~S}$ and p38 MAPK inhibitor block the DOX-induced loss of MMP in H9c2 cells. As it has been demonstrated that ROS-elicited mitochondrial insult participates in DOX-induced cardiotoxicity (20), we investigated the effects of both $\mathrm{H}_{2} \mathrm{~S}$ and p38 MAPK inhibitor on the DOX-induced dissipation of MMP. As shown in Fig. 8, after $\mathrm{H} 9 \mathrm{c} 2$ cells were subjected to $5 \mu \mathrm{M}$ DOX for $24 \mathrm{~h}$, mitochondria were markedly damaged, leading to a decrease in the uptake of JC-1, indicating a loss of MMP (Fig. 8B). Notably, the loss of MMP was ameliorated by pretreatment with $400 \mu \mathrm{M}$ NaHS for $30 \mathrm{~min}$ (Fig. 8D and G). Similarly, pretreatment of $\mathrm{H} 9 \mathrm{c} 2$ cells with $3 \mu \mathrm{M}$ SB203580 for $60 \mathrm{~min}$ before exposure to DOX also blocked the DOX-induced dissipation of MMP (Fig. 8C and G). These data suggest that both exogenous $\mathrm{H}_{2} \mathrm{~S}$ and p38 MAPK inhibitor protect $\mathrm{H} 9 \mathrm{c} 2$ cells against DOX-induced mitochondrial damage. 


\section{Discussion}

Although intensive studies on DOX-induced cardiotoxicity have continued for decades, the underlying mechanisms have not been fully elucidated. Accumulating evidence supports the notion that free radical-induced oxidative stress and cardiomyocyte death by apoptosis and necrosis are the key contributors to DOX-induced cardiotoxicity. In agreement with previous studies $(5-7,11-13,20,22-24)$, in the present study, we observed that exposure of H9c2 cells to DOX markedly induced cellular injuries, including a decrease in cell viability, increases in cell apoptosis, expression of cleaved caspase- 3 and ROS generation as well as dissipation of MMP. We further confirmed that the activation of p38 MAPK plays a pivotal role in DOX-induced cardiotoxicity. This is supported by the findings of this study that i) treatment with $5 \mu \mathrm{M}$ DOX time-dependently upregulated the expression of p-p38 MAPK; ii) pretreatment of H9c2 cells with SB203580, a specific inhibitor of p38 MAPK, dramatically attenuated DOX-induced cytotoxicity, leading to an increase in cell viability; iii) pretreatment with SB203580 markedly reduced the increased number of apoptotic cells and expression level of cleaved caspase-3 induced by DOX; iv) pretreatment with SB203580 obviously decreased DOX-induced ROS generation; v) pretreatment with SB203580 considerably ameliorate DOX-induced dissipation of MMP. All of these results demonstrated the functional significance of the activation of p38 MAPK in DOX-induced cardiotoxicity.

The p38 MAPK is a subfamily of the MAPK superfamily. This subfamily is composed of $\mathrm{p} 38 \alpha, \mathrm{p} 38 \beta, \mathrm{p} 38 \gamma$ and $338 \delta(25-27)$. p38 MAPK has been shown to be a pivotal group of signal molecules that response to environmental stress in various cell types. In cardiomyocytes, p38 MAPK is implicated in the onset of apoptosis in ischemia-reperfusion-injured hearts $(28,29)$. Particularly, transfection experiments using primary cultures of neonatal rat cardiomyocytes further demonstrate the involvement of p38 $\alpha$ in myocyte apoptosis (30). Recently, the roles of p38 MAPK in DOX-induced cardiotoxicity have received attention $(6-8,11,12,31)$. Poizat et al (31) reported that DOX activates $\mathrm{p} 38 \alpha$ and $\mathrm{p} 38 \beta$ which are implicated in the phosphorylation of the transcriptional co-activator p300, which parallels the DOX-induced apoptosis of primary neonatal cardiomyocytes. In transgenic mice containing high levels of cardiac metallothionein (MT) and neonatal mouse cardiomyocytes, the activation of $\mathrm{p} 38$ MAPK has also been shown to participate in DOX-induced cardiomyocyte apoptosis (7). These previous studies $(7,31)$ support the findings of the present study.

Since the common observation is that cardiomyocyte apoptosis contributes to DOX-induced cardiotoxicity, therefore, in the present study, we investigated the possible roles of p38 MAPK in DOX-induced apoptosis. Based on the results obtained from the recent study and a previous observation (31), the following mechanism may be responsible, at least partly, for the roles of p38 MAPK in DOX-induced cardiac apoptosis. Firstly, the activation of p38 MAPK induces accumulation of ROS which was attenuated by pretreatment with SB203580, a specific inhibitor of p38 MAPK. Secondly, p38 MAPK enhanced the activation of cleaved caspase-3, one of the apoptotic effectors. Third, p38 MAPK induced dissipation of MMP. Fourth, p38 MAPK activates p300 (31), which triggers cardiomyocyte apoptosis. Since SB203580 used in the present study acts as a specific inhibitor of $\mathrm{p} 38 \alpha$ and $\mathrm{p} 38 \beta$, but not $\mathrm{p} 38 \gamma$ and $\mathrm{p} 38 \delta$, the exact roles of the different subunits of p38 MAPK require further study. Noteworthy, the results of the present study provide novel evidence that an interaction between ROS and p38 MAPK exists in DOX-treated H9c2 cells, as SB203580 pretreatment attenuated DOX-induced ROS production, whereas the pretreatment of $\mathrm{H} 9 \mathrm{c} 2$ cells with NAC (ROS scavenger) prior to DOX exposure reduced the expression level of p-p38 MAPK. These results are comparable with our previous study (32). More understanding of the role of the interaction between ROS and p38 MAPK in DOX-induced cardiotoxicity may aid in the treatment and prevention of cardiac injury.

Another important novel finding of the present study was that exogenous $\mathrm{H}_{2} \mathrm{~S}$ protects against the DOX-induced cardiotoxicity by inhibition of the p38 MAPK pathway in H9c2 cells. Increasing evidence has shown that $\mathrm{H}_{2} \mathrm{~S}$ is cardioprotective $(13,16-18,33,34)$. Recently, we showed that exogenous $\mathrm{H}_{2} \mathrm{~S}$ offers protection against chemical hypoxia-induced injury by its antioxidant effect and upregulation of heat shock protein 90 (HSP90) expression in H9c2 cells $(18,33)$. Importantly, our more recent study demonstrated that DOX inhibits the expression and activity of cystathionine- $\gamma$-lyase (CSE), an $\mathrm{H}_{2} \mathrm{~S}$ synthase, and that exogenous $\mathrm{H}_{2} \mathrm{~S}$ prevents DOX-induced cardiotoxicity by inhibiting ER stress and oxidative stress (13). Furthermore, we found that the inhibitory effect of exogenous $\mathrm{H}_{2} \mathrm{~S}$ on the activation of p38 MAPK was induced by chemical hypoxia in PC12 cells (19). The results of our recent studies (19) suggest that inhibition of p38 MAPK may contribute to the protective effect of exogenous $\mathrm{H}_{2} \mathrm{~S}$ against DOX-induced cardiotoxicity. The findings of the present study support this hypothesis. We found that pretreatment of H9c2 cells with NaHS (a donor of $\mathrm{H}_{2} \mathrm{~S}$ ) prior to exposure to DOX significantly attenuated the DOX-induced increase in expression of p-p38 MAPK. In addition, pretreatment with NaHS had a similar cardioprotective effect as SB203580 (an inhibitor of p38 MAPK) against DOX-induced cardiotoxicity, as evidenced by an increase in cell viability, and decreases in the number of apoptotic cells, the expression of cleaved caspase-3, dissipation of MMP and ROS accumulation. To explore the mechanism responsible for the inhibitory effect of exogenous $\mathrm{H}_{2} \mathrm{~S}$ on DOX-induced activation of p38 MAPK, $\mathrm{H} 9 \mathrm{c} 2$ cells were pretreated with NAC, an ROS scavenger, before DOX exposure. Similar to exogenous $\mathrm{H}_{2} \mathrm{~S}$, NAC pretreatment also markedly inhibited the increased expression of p-p38 MAPK, suggesting that the inhibitory effect of exogenous $\mathrm{H}_{2} \mathrm{~S}$ on the activation of p38 MAPK may be associated with its antioxidation. Our results are comparable with a previous study that MT having an antioxidative effect decreased DOX-induced cardiomyocyte apoptosis through inhibition of p38 MAPK (7).

In conclusion, in DOX-treated H9c2 cardiac cells, we demonstrated for the first time that exogenous $\mathrm{H}_{2} \mathrm{~S}$ protects against cardiotoxicity by inhibiting the activation of p38 MAPK induced by DOX treatment. Investigation of the potential of $\mathrm{H}_{2} \mathrm{~S}$ to protect against DOX-induced cardiotoxicity 
may lead to the development of novel approaches to this clinical problem.

\section{Acknowledgements}

This study was supported by the Science and Technology Planning Project of Guangdong Province of China (2010B080701035 and 2009B080701014).

\section{References}

1. Ferrans VJ: Overview of cardiac pathology in relation to anthracycline cardiotoxicity. Cancer Treat Rep 62: 955-961, 1978.

2. Hrdina R, Gersl V, Klimtova I, Simunek T, Machackova J and Adamcova M: Anthracycline-induced cardiotoxicity. Acta Medica 43: 75-82, 2000.

3. Scully RE and Lipshultz SE: Anthracycline cardiotoxicity in long-term survivors of childhood cancer. Cardiovasc Toxicol 7: 122-128, 2007.

4. Shan K, Lincoff AM and Young JB: Anthracycline-induced cardiotoxicity. Ann Intern Med 125: 47-58, 1996.

5. Singal PK, Li T, Kumar D, Danelisen I and Iliskovic N: Adriamycin-induced heart failure: mechanism and modulation. Mol Cell Biochem 207: 77-86, 2000.

6. Spallarossa P, Garibaldi S, Altieri P, et al: Carvedilol prevents doxorubicin-induced free radical release and apoptosis in cardiomyocytes in vitro. J Mol Cell Cardiol 37: 837-846, 2004.

7. Kang YJ, Zhou ZX, Wang GW, Buridi A and Klein JB: Suppression by metallothionein of doxorubicin-induced cardiomyocyte apoptosis through inhibition of p38 mitogen-activated protein kinases. J Biol Chem 275: 13690-13698, 2000.

8. Zhu W, Zou Y, Aikawa R, et al: MAPK superfamily plays an important role in daunomycin-induced apoptosis of cardiac myocytes. Circulation 100: 2100-2107, 1999.

9. Sheng Z, Knowlton K, Chen J, Hoshijima M, Brown JH and Chien KR: Cardiotrophin 1 (CT-1) inhibition of cardiac myocyte apoptosis via a mitogen-activated protein kinase-dependent pathway. Divergence from downstream CT-1 signals for myocardial cell hypertrophy. J Biol Chem 272: 5783-5791, 1997.

10. Sugden PH and Clerk A: 'Stress-responsive' mitogen-activated protein kinases (c-Jun $\mathrm{N}$-terminal kinases and p38 mitogenactivated protein kinases) in the myocardium. Circ Res 83: 345-352, 1998

11. Lou H, Kaur K, Sharma AK and Singal PK: Adriamycin-induced oxidative stress, activation of MAP kinases and apoptosis in isolated cardiomyocytes. Pathophysiology 13: 103-109, 2006.

12. Lou H, Danelisen I and Singal PK: Involvement of mitogenactivated protein kinases in adriamycin-induced cardiomyopathy. Am J Physiol Heart Circ Physiol 288: H1925-H1930, 2005.

13. Wang XY, Yang CT, Zheng DD, et al: Hydrogen sulfide protects H9c2 cells against doxorubicin-induced cardiotoxicity through inhibition of endoplasmic reticulum stress. Mol Cell Biochem 363: 419-426, 2012.

14. Lowicka $\mathrm{E}$ and Beltowski J: Hydrogen sulfide $\left(\mathrm{H}_{2} \mathrm{~S}\right)$ - the third gas of interest for pharmacologists. Pharmacol Rep 59: 4-24, 2007.

15. Moore PK, Bhatia M and Moochhala S: Hydrogen sulfide: from the smell of the past to the mediator of the future? Trends Pharmacol Sci 24: 609-611, 2003.

16. Geng B, Chang L, Pan C, et al: Endogenous hydrogen sulfide regulation of myocardial injury induced by isoproterenol. Biochem Biophys Res Commun 318: 756-763, 2004.
17. Wei $\mathrm{H}$, Zhang $\mathrm{R}$, Jin $\mathrm{H}$, et al: Hydrogen sulfide attenuates hyperhomocysteinemia-induced cardiomyocytic endoplasmic reticulum stress in rats. Antioxid Redox Signal 12: 1079-1091, 2010.

18. Yang Z, Yang C, Xiao L, et al: Novel insights into the role of HSP90 in cytoprotection of $\mathrm{H}_{2} \mathrm{~S}$ against chemical hypoxiainduced injury in $\mathrm{H} 9 \mathrm{c} 2$ cardiac myocytes. Int J Mol Med 28: 397-403, 2011.

19. Lan A, Liao X, Mo L, et al: Hydrogen sulfide protects against chemical hypoxia-induced injury by inhibiting ROS-activated ERK1/2 and p38MAPK signaling pathways in PC12 cells. PLoS One 6: e25921, 2011

20. Shi Y, Moon M, Dawood S, McManus B and Liu PP: Mechanisms and management of doxorubicin cardiotoxicity. Herz 36: 296-305, 2011.

21. MacKenzie SH and Clark AC: Death by caspase dimerization. Adv Exp Med Biol 747: 55-73, 2012.

22. Liu J, Mao W, Ding B and Liang CS: ERKs/p53 signal transduction pathway is involved in doxorubicin-induced apoptosis in H9c2 cells and cardiomyocytes. Am J Physiol Heart Circ Physiol 295: H1956-H1965, 2008.

23. Neilan TG, Blake SL, Ichinose F, et al: Disruption of nitric oxide synthase 3 protects against the cardiac injury, dysfunction, and mortality induced by doxorubicin. Circulation 116: 506-514, 2007.

24. Zhang YW, Shi J, Li YJ and Wei L: Cardiomyocyte death in doxorubicin-induced cardiotoxicity. Arch Immunol Ther Exp 57: 435-445, 2009

25. Lechner C, Zahalka MA, Giot JF, Moller NP and Ullrich A: ERK6, a mitogen-activated protein kinase involved in $\mathrm{C} 2 \mathrm{C} 12$ myoblast differentiation. Proc Natl Acad Sci USA 93: 4355-4359, 1996.

26. Lee JC, Laydon JT, McDonnell PC, et al: A protein kinase involved in the regulation of inflammatory cytokine biosynthesis. Nature 372: 739-746, 1994.

27. Young PR, McLaughlin MM, Kumar S, et al: Pyridinyl imidazole inhibitors of p38 mitogen-activated protein kinase bind in the ATP site. J Biol Chem 272: 12116-12121, 1997.

28. Bogoyevitch MA, Gillespie-Brown J, Ketterman AJ, et al: Stimulation of the stress-activated mitogen-activated protein kinase subfamilies in perfused heart. p38/RK mitogen-activated protein kinases and c-Jun $\mathrm{N}$-terminal kinases are activated by ischemia/reperfusion. Circ Res 79: 162-173, 1996.

29. Lin Q, Schwarz J, Bucana C and Olson EN: Control of mouse cardiac morphogenesis and myogenesis by transcription factor MEF2C. Science 276: 1404-1407, 1997.

30. Wang Y, Huang S, Sah VP, et al: Cardiac muscle cell hypertrophy and apoptosis induced by distinct members of the p38 mitogen-activated protein kinase family. J Biol Chem 273: 2161-2168, 1998.

31. Poizat C, Puri PL, Bai Y and Kedes L: Phosphorylationdependent degradation of $\mathrm{p} 300$ by doxorubicin-activated $\mathrm{p} 38$ mitogen-activated protein kinase in cardiac cells. Mol Cell Biol 25: 2673-2687, 2005.

32. Lan AP, Xiao LC, Yang ZL, et al: Interaction between ROS and p38MAPK contributes to chemical hypoxia-induced injuries in PC12 cells. Mol Med Rep 5: 250-255, 2012.

33. Chen SL, Yang CT, Yang ZL, et al: Hydrogen sulphide protects H9c2 cells against chemical hypoxia-induced injury. Clin Exp Pharmacol Physiol 37: 316-321, 2010.

34. Dong XB, Yang CT, Zheng DD, et al: Inhibition of ROS-activated ERK1/2 pathway contributes to the protection of $\mathrm{H}_{2} \mathrm{~S}$ against chemical hypoxia-induced injury in $\mathrm{H} 9 \mathrm{c} 2$ cells. Mol Cell Biochem 362: 149-157, 2012. 